\title{
To disclose or not to disclose psychological problems to GPs
}

Non-detection of common mental disorders in primary care is often interpreted as a reflection of GPs' knowledge and skills. A common approach to dealing with non-detection of mental disorders in primary care is to provide further psychiatric training to improve the rate and accuracy of recognition of mental health disorders. However, the findings from two recent studies on this topic indicate that this strategy is insufficient to ensure young people's psychological problems are not missed.

In this issue, the study by Mauerhofer et al ${ }^{1}$ on help-seeking patterns of young people shows that they are more likely to seek help for psychological problems from an informal source, such as an educational professional, than from a formal healthcare source. They also found that most of the young people who did not seek help for psychological problems had attended primary care at least once during the previous year.

The study by Haller et al in the March issue of the BJGP highlights the multifaceted problem of identifying mental health disorders in young people. ${ }^{2}$ Their results indicated that non-detection of depression and anxiety symptoms in young primary care patients, aged $16-24$ years, is associated with a young person's mental health literacy, healthrelated fears, frequency of consultations, and continuity of care. GP level of training in mental health or adolescent health care was not associated with identification of mental disorder.

The results of both studies challenge the superficial appeal of GP psychiatric training as the sole strategy for improving recognition of mental health problems in primary care. Taken at face value, results suggest that any improvement requires a focus on both patient and relationship factors, and a need to be more careful when concluding that undetected mental disorder in primary care indicates poor quality of care. The studies by Mauerhofer et $a l^{1}$ and Haller et $a l^{2}$ remind us that there are alternative ways of examining this topic that can be applied, for example, when planning studies or developing practice guidelines to improve the quality of health care provided to young people.

Understanding barriers to identifying young people in need of mental health support is a clinical and research priority for primary care. This is because we know little about the barriers and facilitators to detecting mental ill health among this patient group. The implications of undetected and untreated mental health problems in young people are significant, and could have a serious impact on their immediate and long-term future and on increased health service use in adulthood.

Rather than criticising GPs for nondetection of mental health problems in young people, researchers need to clarify why young people do not disclose emotional distress to health professionals. There is a body of research evidence on adult patient non-disclosure of psychological problems and possible reasons. Disclosure of psychological problems can be understood as part of the decision-making process, where patients actively choose whether or not to disclose their concerns to healthcare providers. Several explanations have been offered for the non-disclosure of psychological problems among patients visiting primary care, although the order of importance of barriers remains unclear.

Adult studies on non-disclosure of psychological problems suggest that patients contribute to non-detection by presenting their distress as somatic rather than emotional; ${ }^{3}$ having a tendency to normalise symptoms; ${ }^{4}$ failing to recognise the significance of psychological distress; ${ }^{5}$ or resisting diagnosis through fear of stigma or embarrassment; ${ }^{6}$

A search of the international literature indicated that many of the barriers to disclosure of psychological problems experienced by young people appear to be universal. The reasons young people give for their reluctance to disclose mental health problems include feeling too embarrassed to discuss their problems; ${ }^{7}$ concerns about confidentiality; ${ }^{8}$ insufficient time during consultations to discuss health concerns; ${ }^{7}$ beliefs that emotional symptoms should be dealt with by the individual rather than a health professional, and beliefs that GPs are not a source of mental health support; ;,10 negative views about pharmacological treatments; ${ }^{10}$ unrecognised need for mental health support; 9 and a belief that GPs would not be interested in emotional problems. ${ }^{10,11}$

From what young people have said, they seem to have similar concerns to adults that limit their disclosure of psychological problems to GPs. However, the evidence to support developmentally-appropriate principles of care for young people in primary care settings has been slow to develop. Most studies are descriptive, but what we need are evaluations of changes in practice, particularly within the UK. To demonstrate that new principles for care are appropriate, they need to show that mental health care for young people in primary care settings has improved or that the perceptions of young people have improved. Despite this lack of research, existing work is meaningful for removing barriers to disclosure of psychological problems by young people. The evidence base on non-disclosure of psychological problems in primary care has implications for individual practitioners, medical education, and public education campaigns.

Since disclosure of emotional problems or help seeking by young people may be delayed, GPs can play a key role in facilitating disclosure. Good communication between clinicians and patients is pivotal to treatment, the success of which rests on quality relationships that GPs build with individual patients. But building trusting relationships takes time, which may have implications for consultation length. The 
Royal College of General Practitioners has suggested that consultation length may need to be modified, emphasising the low levels of patient-centredness in UK general practice. ${ }^{12}$

Developing positive, trusting relationships with young people may mean that early attention needs to be given to strengthening communication skills in ways that are appropriate to engaging young people. This reflects a view that forming trusting relationships with young people is best served by nurturing better doctor-patient relationships with them as children. Cahill and Papageorgiou wrote a review on the issue of consulting with children in primary care, and in it they provide a number of useful suggestions for improving consultation behaviour. ${ }^{13}$

Initiatives at international and national levels in response to the growing demand for developmentally-responsive health care highlight the need for health professionals to understand existing barriers to healthcare provision for young people. For example, all UK healthcare professionals who work with young people were recently advised of the need to provide youth-friendly health services, to provide 'access to age-appropriate services which are responsive to their specific needs', and to practice 'effective communication and engagement' with young people. ${ }^{14}$

Awareness of primary care patients' reasons for non-disclosure of psychological problems may be the first step towards understanding the difficulties of detecting mental health problems in young people. This awareness may also show the reasons why encouraging disclosure is challenging for health professionals.
Few studies have investigated the differences between detected and undetected mental disorder in young people in relation to demographic, individual, or clinical variables. The studies by Mauerhofer et $a l^{1}$ and Haller et $a l^{2}$ are a welcome step forward in this effort. They complement recent research showing that more attention needs to be given to what happens within the doctor-patient consultation. The complex interaction is affected by what is said, and by whom, by patient expectations about what might be helpful, and by GPs' and patients' beliefs; all of which are constrained by the limitations on consultation time.

Improving young people's recognition of mental health problems and raising their awareness that GPs are an appropriate source of health care for psychological problems could be addressed by educational campaigns. Taking into account the increasing emphasis on patient and public involvement, there may be a role for young people to assist with the development and evaluation of mental health literacy interventions.

\section{Lynda Tait,}

Research Fellow, School of Health \& Population Sciences, Primary Care Clinical Sciences,

University of Birmingham.

\section{Provenance}

Commissioned; peer reviewed

\section{REFERENCES}

1. Mauerhofer A, Berchtold A, Michaud P-A, Suris J-C GPs' role in the detection of psychological problems of young people: a population-based study. Br J Gen Pract 2009; DOI: 10.3399/bjgp09X454115.

2. Haller DM, Sanci LA, Sawyer SM, Patton GC. The identification of young people's emotional distress: a study in primary care. Br J Gen Pract 2009; DOI: 10.3399/bjgp09X419510.

3. Olde Hartman T, van Rijswijk E, van Ravesteijn H, et al. Mental health problems and the presentation of minor illnesses: data from a 30-year follow-up in general practice. Eur J Gen Pract 2008; 14(Suppl 1): 38-43.

4. Kessler D, Lloyd K, Lewis G, Gray DP. Cross sectional study of symptom attribution and recognition of depression and anxiety in primary care. BMJ 1999; 318(7181): 436-439.

5. Jorm AF. Mental health literacy: public knowledge and beliefs about mental disorders. Br J Psychiatry 2000; 177: 396-401.

6. Outram S, Murphy B, Cockburn J. Factors associated with accessing professional help for psychological distress in midlife Australian women. J Ment Health . 2004; 13(2): 185-195.

7. Churchill R, Allen J, Denman S, et al. Do the attitudes and beliefs of young teenagers towards general practice influence actual consultation behaviour? $\mathrm{Br} J$ of Gen Pract 2000; 50(461): 953-957.

8. Ford CA, Bearman PS, Moody J. Foregone health care among adolescents. JAMA 1999; 282(23): 2227-2234.

9. Bushnell J, McLeod D, Dowell A, et al. Do patients want to disclose psychological problems to GPs? Fam Pract 2005; 22(6): 631-637.

10. Biddle L, Donovan JL, Gunnell D, Sharp D. Young adults' perceptions of GPs as a help source for mental distress: a qualitative study. Br J Gen Pract 2006; 56(533): 924-931.

11. Prior L, Wood F, Lewis G, Pill R. Stigma revisited, disclosure of emotional problems in primary care consultations in Wales. Soc Sci Med 2003; 56(10): 2191-2200

12. Royal College of General Practitioners. The future direction of general practice: A roadmap. London: RCGP, 2007.

13. Cahill P, Papageorgiou A. Triadic communication in the primary care paediatric consultation: a review of the literature. Br J Gen Pract 2007: 57(544): 904-911.

14. Department of Health. Core document, national service framework for children, young people and maternity services. London: Department of Health, 2004.

DOI: 10.3399/bjgp09X454034

\section{ADDRESS FOR CORRESPONDENCE}

\section{Lynda Tait}

University of Birmingham, Primary Care Clinical Sciences, School of Health \& Population Sciences, Primary Care Clinical Sciences Building, Edgbaston Birmingham B15 2TT.

Email: I.tait.1@bham.ac.uk 